\title{
Expanding the pillararene chemistry: synthesis and application of a $10+1$ functionalized pillar[5]arene
}

\author{
Márton Bojtár, ${ }^{\mathrm{a}}$ András Simon, ${ }^{\mathrm{b}}$ Petra Bombicz, ${ }^{\mathrm{c}}$ István Bitter*a \\ ${ }^{a}$ Department of Organic Chemistry and Technology, Budapest University of Technology and Economics, 1521 Budapest, \\ Hungary \\ ${ }^{\mathrm{b}}$ Department of Inorganic and Analytical Chemistry, Budapest University of Technology and Economics, 1521 Budapest, \\ Hungary \\ ${ }^{\mathrm{c}}$ Research Centre for Natural Sciences, Hungarian Academy of Sciences, H-1519 Budapest, Hungary
}

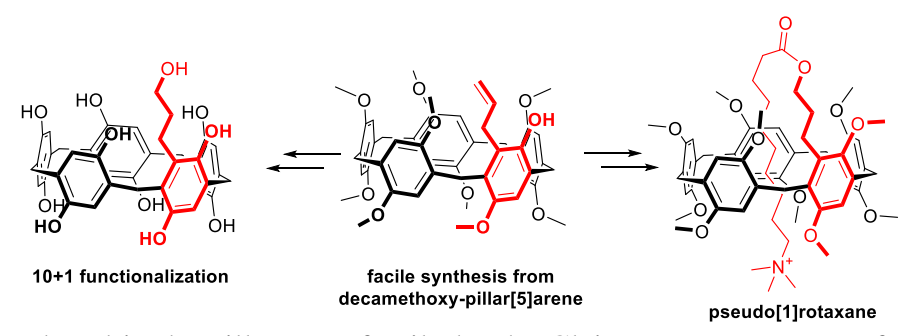

A novel functionality was introduced in the pillararene family by the Claisen rearrangement of monoallyl pillar[5]arene. This new key derivative can lead to the formation of a 10+1 functionalized derivative, a useful scaffold for further derivatization. Except for the previously known monodeprotection reaction, all steps proceed efficiently with high yields and easy separation. In addition, a solvent-responsive pseudo[1] rotaxane was synthesized based on this novel functionality.

Since the appearance of supramolecular chemistry, macrocycles have always had a crucial role in the construction of host-guest systems. Cyclodextrins, ${ }^{1}$ calixarenes, ${ }^{2,3}$ cucurbiturils $^{4,5}$ and other cyclophanes significantly influenced the chemical and related sciences in the past few decades, mainly due to their inherent ability to form supramolecular complexes with various guests. ${ }^{6}$ Pillar $[n]$ arenes are a new class of macrocycles consisting of $n$ hydroquinone units linked in $p$ position with a methylene bridge. ${ }^{7}$ In addition to their unique structural properties for host-guest interactions, their versatile synthetic manipulation offers countless applications based on their tunable molecular recognition. ${ }^{8-13}$ Amongst the pillararene homologues, pillar[5]arene is studied the most due to its high yielding synthesis from inexpensive reagents. In the past few years, great efforts have been made to access differently substituted pillararenes for various purposes. ${ }^{9}$ Most of the work concerns the inherent functionality of the macrocycles, which are the oxygen atoms in the $p$-position of the phenylene rings. Current strategies for functionalizing pillararenes are as follows: (i) deprotection of the methoxy groups to obtain perhydroxylated pillararenes suitable for etherification to yield symmetrical, perfunctionalized hosts ${ }^{7,14-16}$ (ii) cyclization reaction of the functionalized monomer (i. e. bromide) ${ }^{17}$ (iii) deprotection of a single or more methoxy group to yield monoand oligo-functionalized pillararenes ${ }^{18,19}$ (iv) oxidation followed by reduction to yield A1/A2 difunctional or 4- and 6functionalized pillararenes ${ }^{20-22}$ (v) co-cyclization of the monomer containing a functional group for monofunctionalized pillararenes $^{23,24}$ (vi) rim-differentiated pillararenes ${ }^{25,26}$ (vii) phenylene-substituted pillararenes. ${ }^{27,28}$ In all cases, except for the last one, the key players are the alkoxy groups.

Water soluble pillararenes are an emerging class of functionalized pillararenes, ${ }^{15,17,29}$ due to their substitutiondependent ability to form host-guest complexes with various important guests. ${ }^{30,31}$ However, to develop chemosensing platforms based on these hosts, the only option is the indicator displacement method since currently no direct syntheses are available for water soluble pillararenes functionalized by a signaling group. ${ }^{32-35}$ Aqueous solubility is often reached by the perfunctionalizaton of the aryloxy groups, therefore the only option for the introduction of a signaling group is the functionalization of a phenylene ring.

As a continuation of our research concerning the supramolecular analytical application of these macrocycles, ${ }^{32,33,36}$ we developed a synthetic strategy for the $10+1$ functionalization of pillar[5]arene based on the Claisen rearrangement reaction which was previously used for the phenylene functionalization of calixarenes. ${ }^{37}$ Starting from monohydroxy-pillar[5]arene, a direct and effective synthesis can be performed to obtain a phenylene-substituted derivative with the possibility of further functionalization of the phenylene oxygens and this new functionality, separately. In this paper, the synthesis, characterization and crystal structure of of 3-allyl-4hydroxy-nonamethoxy-pillar[5]arene (3) is described. In addition, we report a novel solvent-responsive pseudo[1]rotaxane based on this scaffold. 
Scheme 1 Synthesis of monoallyl monohydroxy pillar[5]arene 3

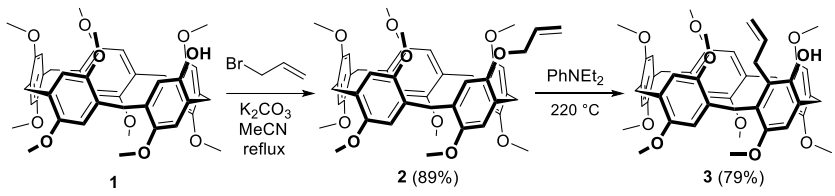

The starting material monohydroxy-pillar[5]arene was synthesized as described with some modifications (see ESI for details). ${ }^{18,19}$ The monoallyl derivative $\mathbf{2}$ was obtained in high yield in a simple alkylation reaction without cromatography (Scheme 1). The Claisen rearrangement proceeded smoothly at $220^{\circ} \mathrm{C}$ in $N, N$-diethylaniline similarly as reported in the case of calixarenes, however in higher yield (79\% compared to $60 \%){ }^{37}$ As monitored by TLC, a reaction time of 6 hours is required for the completion of the rearrangement. The compounds were characterized by NMR and HRMS methods. The ${ }^{1} \mathrm{H}-\mathrm{NMR}$ spectrum of $\mathbf{3}$ can be seen of Figure 1.

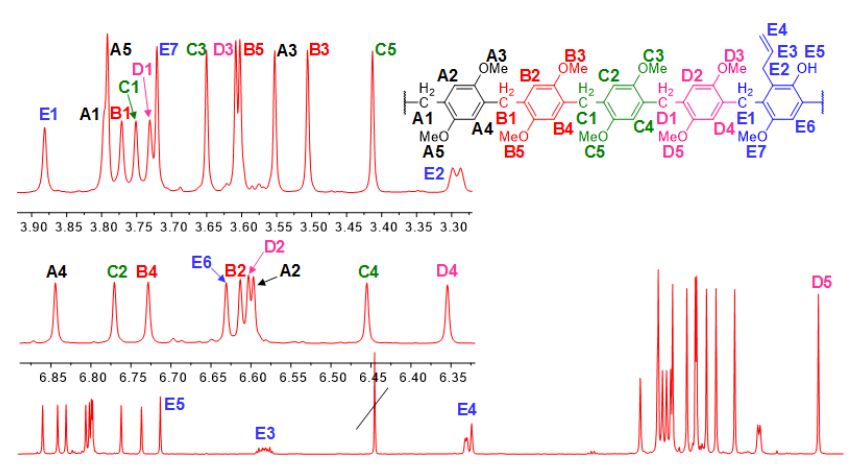

Figure $1{ }^{1} \mathrm{H}-\mathrm{NMR}\left(500 \mathrm{MHz}, \mathrm{CDCl}_{3}\right)$ spectrum of 3

The crystallization of $C$-monoallyl-monohydroxypillar[5]arene (3) was tried in different solvents. It was possible to determine the structure of the acetone inclusion compound of 3 by single crystal X-ray diffraction. ${ }^{38}$ The non-symmetric substitution of the pillar[5] arene reduces the symmetry of the crystal to $C 2 / c$. The stoichiometric ratio of $\mathbf{3}$ to acetone is 1 to 1.5. One of the acetone is placed in the cavity of the pillar[5] arene, while that situated in a twofold axis can be found among the host molecules. The host molecules turn out of the column formed along the $\mathrm{b}$ crystallographic axis with their apertures, therefore no channels but individual voids accommodate the acetone guest molecules. As the result of allyl substitution one of the neighbouring phenylene rings turns upside down (Figure 2). It makes possible the formation of a hydrogen bond between their substituents, the unprotected $\mathrm{OH}$ group and the methoxy oxygen, contributing to the stability of the pillar[5] arene molecule. ${ }^{39}$

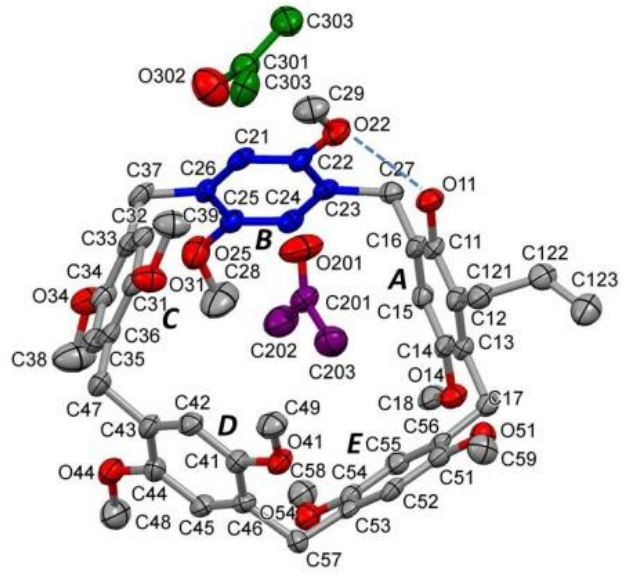

Figure 2 The ORTEP representation of $\mathbf{3}$ with acetone inclusion at $50 \%$ probability level. Hydrogen atoms are omitted for clarity. The carbon atoms of the acetone molecule placed in the cavity are colored purple, while the carbons of the acetone can be found among the host molecules are green. Ring $\mathrm{A}$ is the substituted phenylene ring, ring B (highlighted in blue) is turned upside down in order to form a hydrogen bond.

Scheme 2 Synthesis of the 10+1 functionalized pillar[5]arene 6

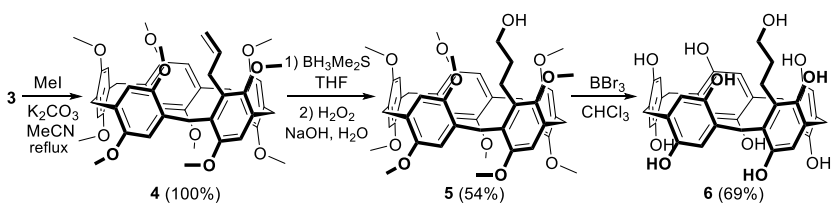

The further functionalization of $\mathbf{3}$ is presented in Scheme 2. First, the protection of the hydroxyl group was established with a simple methylation with a large excess of methyl iodide to obtain $C$-allyl-decamethoxy-pillar[5]arene (4) quantitatively. One of the simplest derivatization of an allylic group is the hydroboration-oxidation reaction to obtain the $\omega$-hydroxyl derivative. First, 4 was reacted with borane-dimethylsulfide in THF to form the borane compound, then the oxidation step was performed with the usual alkaline peroxide reagent. After cromatographic separation, a moderate yield of $54 \%$ was achieved, and some of the starting material was recovered. Unfortunately, higher borane excess did not lead to utter conversion. The separation in this reaction and in each step is facile since the polarity of the compunds change due to the manipulation with $\mathrm{OH}$ groups. This new functionality is resistant to boron tribromide, therefore the total deprotection of the methoxy groups should lead to the desired 10+1 functional pillar[5] arene. Using a large excess of $\mathrm{BBr}_{3}$, we obtained 6 in good yield $(69 \%)$. As in the case of parent decahydroxypillar[5]arene, this compund can also undergo oxidation on air, therefore it is advisable to store under water or aqueous thiosulfate to prevent the formation of a brown product. All of these compunds were characterised by NMR and HRMS techniques. The scaling up of this reaction is now underway in our laboratory with the aim of the functionalization of the water soluble derivatives. We are currently working on the further derivatization of the allylic or hydroxy group (i.e. oxidation and substitution reactions) to futher extend the application of this novel functionality. 
Scheme 3 Synthesis of the monofunctionalized ester 7

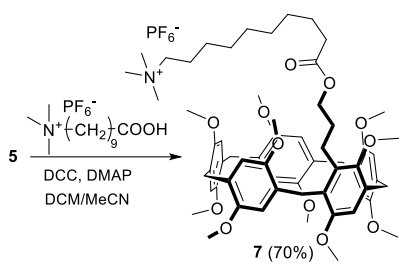

The different functionalization compared to the $O$-alkyl derivatives could not only lead to novel synthetic targets but to construct interesting supramolecular structures based on the versatile host-guest properties of this macrocycle family. Although some pillararene-based pseudo[1]rotaxanes exist, the axle part of the molecule is always connected via $O$ substitution. ${ }^{40-44}$ To demonstrate the applicability of this new functionality, we have designed $\mathbf{7}$ in the hope of investigating its self-inclusion properties The hydroxypropyl derivative was easily acylated by 10 -trimethylammonio-decanoic acid to yield the respective ester 7 (Scheme 3). Note that due to the higher rigidity of the structure compared to $O$-alkylated derivatives, we incorporated a rather long aliphatic chain with the complexing trimethylammonium unit at the terminal position. The chain length from the macrocycle to the cationic group is 14 atoms that enables high conformational freedom for self-inclusion.

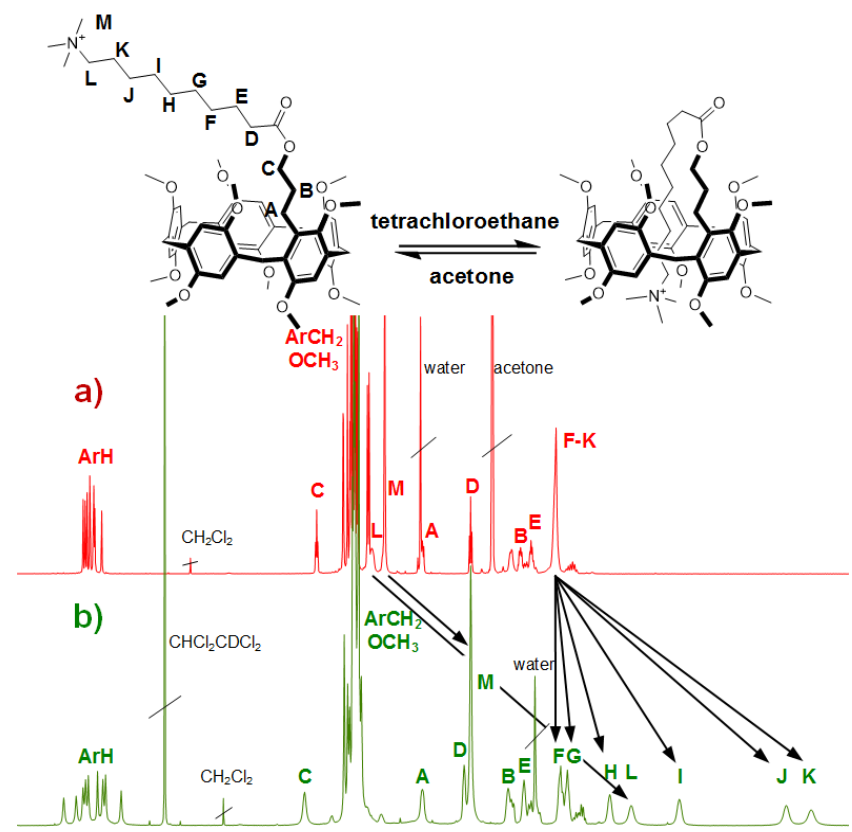

Figure $3{ }^{1} \mathrm{H}-\mathrm{NMR}$ spectra of 7 in a) acetone-d6 and b) tetrachloroethane- $d_{2}\left(25{ }^{\circ} \mathrm{C}, 500 \mathrm{MHz}, 15 \mathrm{mM}\right.$ concentration in each case). The assignation was provided from COSY and HMBC experiments, the arrows indicate the shifting of the signals due to self-complexation

The ${ }^{1} \mathrm{H}-\mathrm{NMR}$ spectrum of pillararene 7 in acetone- $d_{6}$ shows no sign of self-complexation. However, when the solvent was replaced to chloroform, large alteration in the spectrum was observed. The spectrum in $\mathrm{CDCl}_{3}$ was, however of poor quality due to the high conformational instability of 7 . This was overcome by using tetrachloroethane- $d_{2}$ as NMR-solvent. Tetrachloroethane is considerably larger than the cavity of the macrocycle, therefore we estimated that this particular solvent is ideal for driving the side-chain into the cavity. The ${ }^{1} \mathrm{H}-\mathrm{NMR}$ spectra are shown in Figure 3. The assignation of the side-chain is provided from the COSY spectra (Figures S27 and S31 in the Supporting information) in each solvent. According to the shifts in the resonances of the side-chain protons and the ROESY correlations (Figure S32 in the Supporting information), the trimethyl-ammonium group is located at the lower portal of the cavity, and the double bend in the chain is formed near the ester group. To prove self-inclusion instead of supramolecular polymer formation, dilution of the sample was performed. Upon dilution, the supramolecular complexes should dissociate due to the lower concentration which would result in the shifts of the resonances. As can be seen in Figure S33 in the Supporting information, no deviation was observed within more than 2 orders of magnitude of concentration (from $42 \mathrm{mM}$ to 0.234 $\mathrm{mM}$ ), which indicate indeed the pseudo[1] rotaxane nature of 7 . We have also investigated the addition of small amounts of acetone- $d_{6}$ to the tetrachloroethane- $d_{2}$ solution of 7 (Figure S34). Upon addition of more than 25 equivalents of acetone, the signals started to deviate that can be rationalized by the equilibrium of the self-complexed and open form.

In conclusion, we have introduced a novel functionality in the pillararene family by the Claisen rearrangement of the monoallyl derivative. This can lead to the formation of a $10+1$ functionalized derivative, decahydroxy-monohydroxypropilpillar[5]arene which should be a key intermediate towards multi-functionalized pillar[5]arenes, i. e. functionalized watersoluble molecules. Except for the previously known monodeprotection reaction, all steps proceed efficiently with high yields and easy separation. In addition, we synthesized by esterification a pseudo[1]rotaxane, that shows solventdependent self-complexation. We believe that this novel synthesis could be an ideal platform to construct previously unknown pillararene derivatives to further extend their remarkable applications.

\section{ASSOCIATED CONTENT}

Supporting Information. Materials and methods, synthetic procedures, characterization data, NMR spectra and crystallographic information (PDF). Single-crystal X-ray data for 3 (CIF).

\section{AUTHOR INFORMATION}

Corresponding author

*Email: bitter@oct.bme.hu

\section{ACKNOWLEDGEMENTS}

We thank the Hungarian Scientific Research Fund for the financial support of this work (Grant No. K-108752). P. B. is grateful for the Hungarian Scientific Research Fund (K124544).

\section{REFERENCES}

(1) Crini, G. Chem. Rev. 2014, 114 (21), 10940.

(2) Böhmer, V. Angew. Chemie Int. Ed. English 1995, 34 (7),

(3) Nimse, S. B.; Kim, T. Chem. Soc. Rev. 2013, 42 (1), 366.

(4) Lagona, J.; Mukhopadhyay, P.; Chakrabarti, S.; Isaacs, L. Angew. Chem., Int. Ed. 2005, 44 (31), 4844.

(5) Barrow, S. J.; Kasera, S.; Rowland, M. J.; Del Barrio, J.; Scherman, O. A. Chem. Rev. 2015, 115 (22), 12320.

(6) Liu, Z.; Nalluri, S. K. M.; Stoddart, J. F. Chem. Soc. Rev. 2017, 46 (9), 2459

(7) Ogoshi, T.; Kanai, S.; Fujinami, S.; Yamagishi, T.; Nakamoto, Y. J. Am. Chem. Soc. 2008, 130 (15), 5022.

(8) Ogoshi, T.; Yamagishi, T.; Nakamoto, Y. Chem. Rev. 2016, $116(14), 7937$. 
(9) Strutt, N. L.; Zhang, H.; Schneebeli, S. T.; Stoddart, J. F. Acc. Chem. Res. 2014, 47 (8), 2631.

(10) Xue, M.; Yang, Y.; Chi, X.; Zhang, Z.; Huang, F. Acc. Chem. Res. 2012, 45 (8), 1294.

(11) Cragg, P. J.; Sharma, K. Chem. Soc. Rev. 2012, 41 (2), 597.

(12) Zhang, H.; Zhao, Y. Chem. - Eur. J. 2013, 19 (50), 16862.

(13) Yang, K.; Pei, Y.; Wen, J.; Pei, Z. Chem. Commun. 2016, 52 (60), 9316.

(14) Ogoshi, T.; Aoki, T.; Kitajima, K.; Fujinami, S.; Yamagishi, T.; Nakamoto, Y. J. Org. Chem. 2011, 76 (1), 328.

(15) Ogoshi, T.; Hashizume, M.; Yamagishi, T.; Nakamoto, Y. Chem. Commun. 2010, 46 (21), 3708.

(16) Ma, Y.; Chi, X.; Yan, X.; Liu, J.; Yao, Y.; Chen, W.; Huang, F.; Hou, J.-L. Org. Lett. 2012, 14 (6), 1532.

(17) Ma, Y.; Ji, X.; Xiang, F.; Chi, X.; Han, C.; He, J.; Abliz, Z.; Chen, W.; Huang, F. Chem. Commun. 2011, 47 (45), 12340.

(18) Han, J.; Hou, X.; Ke, C.; Zhang, H.; Strutt, N. L.; Stern, C. L.; Stoddart, J. F. Org. Lett. 2015, 17 (13), 3260.

(19) Ogoshi, T.; Demachi, K.; Kitajima, K.; Yamagishi, T. Chem. Commun. 2011, 47 (25), 7164.

(20) Shurpik, D. N.; Padnya, P. L.; Makhmutova, L. I.; Yakimova, L. S.; Stoikov, I. I. New J. Chem. 2015, 39 (12), 9215.

(21) Han, C.; Zhang, Z.; Yu, G.; Huang, F. Chem. Commun. 2012, 48 (79), 9876.

(22) Ogoshi, T.; Yamafuji, D.; Kotera, D.; Aoki, T.; Fujinami, S.; Yamagishi, T. J. Org. Chem. 2012, 77 (24), 11146.

(23) Liu, L.; Cao, D.; Jin, Y.; Tao, H.; Kou, Y.; Meier, H. Org. Biomol. Chem. 2011, 9 (20), 7007.

(24) Strutt, N. L.; Forgan, R. S.; Spruell, J. M.; Botros, Y. Y.; Stoddart, J. F. J. Am. Chem. Soc. 2011, 133 (15), 5668.

(25) Yao, Y.; Xue, M.; Chen, J.; Zhang, M.; Huang, F. J. Am. Chem. Soc. 2012, 134 (38), 15712.

(26) Zhang, Z.; Luo, Y.; Xia, B.; Han, C.; Yu, Y.; Chen, X.; Huang, F. Chem. Commun. 2011, 47 (8), 2417.

(27) Strutt, N. L.; Schneebeli, S. T.; Stoddart, J. F. Supramol. Chem. 2013, 25 (9-11), 596.

(28) Strutt, N. L.; Zhang, H.; Schneebeli, S. T.; Stoddart, J. F. Chem. - Eur. J. 2014, 20 (35), 10996.

(29) Yu, G.; Xue, M.; Zhang, Z.; Li, J.; Han, C.; Huang, F. J. Am. Chem. Soc. 2012, 134 (32), 13248.

(30) Yu, G.; Zhou, J.; Shen, J.; Tang, G.; Huang, F. Chem. Sci. 2016, 7 (7), 4073.

(31) Li, C. J.; Ma, J. W.; Zhao, L.; Zhang, Y. Y.; Yu, Y. H.; Shu,

X. Y.; Li, J.; Jia, X. S. Chem. Commun. 2013, 49 (19), 1924.

(32) Bojtár, M.; Szakács, Z.; Hessz, D.; Kubinyi, M.; Bitter, I. RSC Adv. 2015, 5 (34), 26504.

(33) Bojtár, M.; Paudics, A.; Hessz, D.; Kubinyi, M.; Bitter, I. RSC Adv. 2016, 6 (89), 86269 .

(34) Hua, B.; Shao, L.; Yu, G.; Huang, F. Chem. Commun. 2016 $52(65), 10016$.

(35) Wang, P.; Yao, Y.; Xue, M. Chem. Commun. 2014, 50 (39), 5064

(36) Bojtár, M.; Kozma, J.; Szakács, Z.; Hessz, D.; Kubinyi, M.; Bitter, I. Sens. Actuators, B 2017, 248, 305.

(37) Gutsche, C. D.; Lin, L.-G. Tetrahedron 1986, 42 (6), 1633.

(38) Crystallographic data for the crystal structure of the acetone inclusion of 3 have been deposited with the Cambridge Crystallographic Data Centre as supplementary publication number CCDC 1560837

(39) Resnati, G.; Boldyreva, E.; Bombicz, P.; Kawano, M. IUCrJ 2015, 2 (6), 675

(40) Chen, Y.; Cao, D.; Wang, L.; He, M.; Zhou, L.; Schollmeyer, D.; Meier, H. Chem. - Eur. J. 2013, 19 (22), 7064.

(41) Chen, L.; Li, Z.; Chen, Z.; Hou, J.-L. Org. Biomol. Chem. 2013, 11 (2), 248.

(42) Guan, Y.; Liu, P.; Deng, C.; Ni, M.; Xiong, S.; Lin, C.; Hu, X.-Y.; Ma, J.; Wang, L. Org. Biomol. Chem. 2014, 12 (7), 1079.

(43) Wu, X.; Ni, M.; Xia, W.; Hu, X.-Y.; Wang, L. Org. Chem. Front. 2015, 2 (9), 1013.

(44) Wu, X.; Gao, L.; Sun, J.; Hu, X.-Y.; Wang, L. Chinese Chem. Lett. 2016, 27 (11), 1655. 